\title{
RECHERCHES EXPÉRIMENTALES SUR LA TRICHINOSE
}

\section{DES VOLAILLES}

\author{
Par P. PAVLOV
}

Trichinella spiralis est connu comme un parasite à grand nombre d'hôtes, notamment des carnivores, à cause de son mode de propagation. Cependant, un certain nombre d'herbivores, par voie expérimentale, peuvent, eux aussi, être contaminés.

Il en est autrement des oiseaux. Les expériences que l'on a faites sur eux, et qui consistent en invasion musculaire et intestinale, n'ont pas toujours été réussies. $\mathrm{Si}$ un certain résultat a été obtenu, il n'a jamais été satisfaisant, ou bien il représente une variation des cas que l'on observe chez les carnivores et les herbivores.

De tout ce que nous savons jusqu'à présent sur ce sujet, il appert que, chez les poules, oies, canards et pigeons, on a vraiment pu constater la présence de trichines intestinales, mais les trichines musculaires y manquent absolument. Ces résultats ont donné lieu à croire que les oiseaux possèdent une immunité naturelle contre la trichinose musculaire et que cette immunité est permanente et même absolue. Ces premières constatations ont eu lieu avant que la question ait été résolue par Doerr et ses collaborateurs.

Doerr et Schmidt ont obtenu des résultats positifs dans leurs expériences sur des animaux de laboratoire, consistant en inoculation intramusculaire de trichines femelles adultes ou de jeunes trichines musculaires.

Leurs expériences faites sur des poules ont donné le résultat suivant :

$1^{\circ}$ Dans les cas d'injection de jeunes trichines musculaires dans le muscle pectoral, le résultat a été négatif.

$2^{\circ}$ Dans les cas d'injection de trichines femelles intestinales, adultes, dans le même muscle, le résultat a été positif chez 12 poules sur 19 injectées. Ce résultat permet un changement dans les idées qu'on avait jusqu'alors là-dessus, en ce sens que Doerr et Schmidt ont prouvé que la résistance des oiseaux à la trichinose musculaire est due, non point au tissu musculaire, mais à d'autres causes qui, à leur sens, sont : ou bien la mort des embryons

Annales de Parasitologie, T. XV, $\mathrm{N}^{\circ} 5 .-1^{\text {er }}$ septembre 1937 , p. 434-439. 
dans le sang, ou bien l'impossibilité dans laquelle se trouvent ces derniers de quitter les réseaux capillaires ou de pénétrer dans la circulation de l'organisme.

Notre but étant de contribuer à la solution des questions ainsi posées, nous avons entrepris des expériences d'infestation d'oiseaux en les nourrissant de trichines musculaires. Par ces expériences, nous avons tâché de vérifier l'immunité des oiseaux en cas d'invasion musculaire et intestinale par Trichinella spiralis. En vue de ces recherches, nous avons mis d'abord lesdits oiseaux dans des conditions de vie normales, puis dans des conditions de vie particulières ; nous n רus sommes proposé d'étudier le rôle de la faim, de la température da corps, de l'âge, du régime de nutrition et de l'augmentation arti.jcielle du calcium dans l'organisme, en un mot, nous avons tâché d'étudier l'influence de tous ces facteurs sur l'immunité de ces organismes contre l'infestation par les parasites en question.

Les expériences ont été effectuées sur des pigeons aussi bien que sur des corneilles et des pies, à cause du régime alimentaire différent de ces dernières, comparativement à celui des pigeons.

Dans tous les cas, l'invasion a eu lieu per os et a consisté en introduction de trichines musculaires dans le corps.

Voici le détail de nos expériences :

1. Les premières avaient pour but des recherches sur la résistance des oiseaux à la trichinose musculaire et intestinale loṛsque les conditions de vie chez ceux-ci sont absolument normales. Sept pigeons adụltes, du même âge, avaient pris, le 21-3-1936, en masse, de la viande envahie de Trichinella. Ensuite, ils avaient été remis dans des conditions de vie normale et nourris comme auparavant, c'est-à-dire comme ils l'étaient avant les expériences.

a) L'examen des pigeons traités de la sorte fut commencé le dixième jour après l'ingestion de la viande trichinée ; trois d'entre eux ont été examinés pour les trichines intestinales. Tous les deux jours à partir du $10^{\circ}$, on sacrifiait un pigeon. Chez celui qui avait été sacrifié le $10^{\circ}$ jour après l'ingestion de viande trichinée, le -ésultat fut négatif, en ce qui concerne la trichinose intestinale. Chez celui sacrifié le $12^{\circ}$ jour, à partir de la même date, nous avons trouvé trois Trichinella adultes contenant des embryons bien développés. Quant au pigeon tué le $14^{\circ}$ jour, à partir de la même date, nous avons trouvé cinq trichines avec des embryons bien développés.

b) Les autres pigeons, au nombre de 4 , nourris de Trichinella spiralis en masse, avaient été sacrifiés le 27-4-1936. Nous avons 
examiné la musculature de la tête, du cou, du thorax, des ailes, des pattes et une partie de celle du dos et de la queue. Les museles de la tête et du cou ont été examinés en entier, ceux des autres parties du corps partiellement avec $350-400$ préparations au compresseur pour chaque partie. Toutes ces recherches ont été positives, ce que prouve la découverte d'une Trichinella spiralis dans la musculature de la tête, spiralée, relativement mince, aux extrémités arquées, s'agitant librement dans le suc musculaire. Le résultat des recherches sur les autres pigeons, auxquelles nous avons procédé de la même façon, a été absolument négatif.

c) Dans le même ordre de recherches, nous avons examiné trois petits pigeons incapables de se nourrir seuls. Le 27-4-1936, ils avaient pris en masse des trichines musculaires per os, et avaient été, immédiatement après, remis dans des conditions de vie normales jusqu'au 29-5-1936. La musculature en a été examinée à cette date, tout à fait de la manière dont l'avait été celle des pigeons précédents. Nous n'y avons trouvé qu'une seule Trichinella spiralis : elle était, dans le suc des muscles, entre les fibres musculaires du cou, tout à fait semblable à celle qu'on avait trouvée lors des expériences précédentes.

d) Toujours dans le même but, le 28-3-1936, deux jeunes pigeons, qui étaient cette fois en état de se nourrir seuls, avaient pris per os des Trichinella spiralis en masse avec de la viande. L'un d'eux a été tué et examiné le 12-4-36, pour des trichines intestinales. Il y en avait deux, toutes deux contenant des embryons bien développés. Le suivant a été tué et examiné un mois après l'ingestion. Nous n'avons pas trouvé, chez ce pigeon, dans la musculature, de Trichinella spiralis.

2. La seconde série de recherches a été consacrée à l'examen du rôle de la température du corps lors d'une invasion de Trichinella spiralis, chez les oiseaux nourris en masse de jeunes trichines musculaires. Cinq pigeons de même âge furent installés à partir du 21-4-1936, pendant cinq jours, dans un récipient contenant de l'eau et ensuite nourris en masse de trichines musculaires. Sortis de l'eau pour l'ingestion, ils y avaient été remis immédiatement après et y étaient restés jusqu'à la fin des expériences. La température desdits pigeons avait sensiblement baissé.

L'un deux a été tué et examiné le 25-5-1936 ; la musculature de la tête et du cou a été examinée en entier, tandis que celle du poitrail, des ailes et des pattes, ainsi que le reste du corps, par des préparations « $\mathrm{K}$ », 400 pour chaque organe. On a constaté la présence de deux Trichinella dans le suc musculaire, entre les 
fibres, l'une dans la tête et l'autre dans le cou. Elles n'étaient pas spiralées et leurs extrémités ne l'étaient pas non plus. Par contre, on n'en a pas constaté chez les quatre suivants, examinés successivement tous les trois jours.

3. Il fallait connaitre ensuite l'influence de la faim et de l'inanition de l'organisme sur sa résistance à $T$. spiralis.

Six pigeons avaient été soumis à nos expériences. Après deux jours de jeûne, ils avaient été nourris en masse de trichines musculaires le 15-5-1936. Dans la suite, on leur avait fait à nouveau supporter un manque absolu de nourriture pendant trois jours. Après ce régime, ils avaient été replacés dans leurs conditions de vie normale. Tous ont été examinés du 10 au 20-6-1936. Il n'y a eu, lors de cette exploration, qu'une seule trichine dans les muscles du cou d'un des pigeons, mais pas suffisamment développée.

4. Cinq pigeons, à peu près du même âge, ont été l'objet de nos expériences ultérieures sur l'influence du régime alimentaire sur l'organisme attaqué par Trichinella spiralis, question que nous nous étions proposé d'étudier. Les pigeons en question avaient été nourris exclusivement de viande pendant cinq jours. A la fin du dernier jour, le régime avait été modifié de sorte que la nourriture des pigeons consistait exclusivement en viande envahie en masse de $T$. spiralis. De nouveau, trois jours d'alimentation par de la viande et les pigeons reprirent leur nourriture normale.

Après avoir ingéré de la viande trichinée, deux de ces pigeons avaient été mis dans l'eau et y étaient restés jusqu'à la fin des recherches. Nous avons procédé à l'examen et, 35-40 jours après l'infestation, les parties du corps ont été examinées comme auparavant. La musculature de la tête et du cou a été examinée en détail, tandis que celle du thorax, des pattes, des ailes, aussi bien que celle du reste du corps, par 350 préparations « $K$ ». Un seul des pigeons, mis dans l'eau et examiné 36 jours après son invasion par Trichinella spiralis, avait des trichines au nombre de deux dans la musculature dt cou. Elles n'étaient pas non plus spiralées et se tenaient logées, plus exactement, entre les fibres des muscles.

5. Cette expérience a été effectuée sur deux corneilles et deux jeunes pies, dans le seul but de voir quelle est leur réceptivité envers Trichinella spiralis, étant donné que leur manière de vivre et de se nourrir diffère sensiblement de celle des pigeons.

Après l'ingestion de viande envahie de jeunes trichines en masse, nous avons mis deux d'entre eux, un de chaque espèce, dans des récipients remplis d'eau, de manière que le bas des pattes fût constamment mouillé. Les autres n'avaient été soumis à aucun 
régime spécial. Un mois après l'ingestion, ils avaient été examinés de la manière décrite plus haut. Les préparations avaient été au nombre de 400 pour chaque partie mentionnée chez les autres oiseaux. Le cou et les muscles de la tête ont tous été examinés. Nous avons trouvé une seule trichine dans les muscles de la tête d'une des pies. Cette trichine n'était pas spiralée.

6. Dans cette dernière expérience, nous avons tenté d'augmenter la quantité du calcium dans l'organisme par son augmentation dans l'eau et dans la nourriture, et encore par des injections sous-cutanées de solution de calcium à 1 p. 100 , pendant 15 jours. Cinq pigeons avaient été nourris, en masse, deux fois à un intervalle de deux jours, avec de la viande contenant en abondance des jeunes trichines. Les pigeons ont subi aussi, 25 jours après l'invasion, des injections sous-cutanées de $0 \mathrm{ccm}$., 1 de solution de calcium à 1 p. 100 , pendant 15 jours. Les recherches ont été faites deux mois après, pendant 10 jours. Dans les muscles de la tête, chez un, et dans ceux du cou chez un autre, nous avons trouvé deux trichines insuffisamment développées. De chaque partie du corps, mentionnée dans les recherches plus haut, nous avons fait 400 préparations « $K$ » (sous le trichinoscope). Les muscles du cou et de la tête ont tous été examinés.

\section{Conclusions}

1. Les pigeons, les pies et les corneilles ne possèdent qu'une immunité relative contre la trichinose musculaire et intestinale.

2. Les trichines développées chez eux, comparées à celles de la souris, du rat, du lapin et du cobaye, développées dans la même période de temps, sont plus grêles, non spiralées et sans capsules.

3. Chez les oiseaux, les lieux de prédilection pour Trichinella spiralis sont plus particulièrement les muscles de la tête et du cou, ce qui n'est pas le cas pour les mammifères.

4. Le calcium, en grande quantité dans l'organisme, joue peutêtre un rôle pour la formation des capsules, mais l'absence de ces dernières dans les cas cités jusqu'à présent est due, à notre sens, plutôt à la texture serrée des fibres musculaires, qui empêchent également peut-être la disposition en spirale de Trichinella spiralis.

5. L'abaissement de la température de l'organisme, la faim, le régime alimentaire n'exercent, à notre sens, aucune influence marquée sur l'invasion musculaire des oiseaux par Trichinella spiralis. 


\section{BIBLIOGRAPHIE}

Berger-Staechlin. - Studien über den Mechanismus der Trichinelleninfektion. - III. Mitteilung. Die parenterale trichinige Muskelinfektion. Centrbl. $f$. Bakter., Orig., CVII, H. 6/7, p. 377.

- Studien über den Mechanismus der Trichinelleninfektion. - V. Mitteilung. Weitere Beiträge zur parenteralen trichinigen Muskelinfektion. Centrbl. f. Bakter., Orig., CXI.

Braun-Seifert. - Die tierischen Parasiten des Menschen, I Teil, 1925.

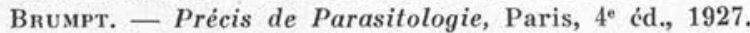

Doerr-SchmidT. - Studien über den Mechanismus der Trichinelleninfektion. VII. Mitteilung. Experimentelle Beinfressung der Trichinenwanderung. - Die natuerliche Immunitaet des Huhnes. Centrbl. f. bak., orig., CXV, p. 427 .

Gruber. - Trichinellen, Trichinosen und ihre Abwehr. Ergebn. d. Hygiene, VIII, 1926 , p. 165.

Ostertag (V.). - Lehrbuch der Fleischbeschau, 7 u. 8. Auflage, 1923.

Institut Vétérinaire Bactériologique pour la Bulgarie du Nord, Tirnovo (Directeur : $D^{\text {r }}$ Chr. Rousseff). 